\title{
Vehicular Ad Hoc Networks
}

\section{Syed R. Rizvi, ${ }^{1}$ Stephan Olariu, ${ }^{1}$ Cristina M. Pinotti, ${ }^{2}$ Shaharuddin Salleh, ${ }^{3}$ Mona E. Rizvi, ${ }^{4}$ and Zainab Zaidi ${ }^{5}$}

\author{
${ }^{1}$ Old Dominion University, Norfolk, VA 23529, USA \\ ${ }^{2}$ Department of Computer Science, University of Perugia, 06123 Perugia, Italy \\ ${ }^{3}$ University of Technology, 81310 Johor, Malaysia \\ ${ }^{4}$ Norfolk State University, Norfolk, VA 23504, USA \\ ${ }^{5}$ NICTA, Australia
}

Correspondence should be addressed to Syed R. Rizvi, srizvi@cs.odu.edu

Received 24 March 2011; Accepted 24 March 2011

Copyright (c) 2011 Syed R. Rizvi et al. This is an open access article distributed under the Creative Commons Attribution License, which permits unrestricted use, distribution, and reproduction in any medium, provided the original work is properly cited.

Vehicular ad hoc networks (VANETs) have recently been proposed as one of the promising ad hoc networking techniques that can provide both drivers and passengers with a safe and enjoyable driving experience. VANETs can be used for many applications with vehicle-to-vehicle $(\mathrm{V} 2 \mathrm{~V})$ and vehicle-toinfrastructure (V2I) communications. In the United States, motor vehicle traffic crashes are the leading cause of death for all motorists between two and thirty-four years of age. In 2009, the National Highway Traffic Safety Administration (NHTSA) reported that 33,808 people were killed in motor vehicle traffic crashes. The US Department of Transportation (US-DOT) estimates that over half of all congestion events are caused by highway incidents rather than by rush-hour traffic in big cities. The US-DOT also notes that in a single year, congested highways due to traffic incidents cost over $\$ 75$ billion in lost worker productivity and over 8.4 billion gallons of fuel. Some of the significant applications of VANETs are road safety applications including collision and other safety warning systems, driver convenience and information systems, and, in the future, intelligent traffic management systems.

This special issue on VANETs presents current groundbreaking research, projects, and standardization efforts that have been done in the area of vehicular communications. In both the review process, and the production process we have aimed for the highest possible quality and speed. Papers in this special issue have been rigorously peer-reviewed. With an internationally acclaimed Editorial Board, we have selected some high-quality research work in the field of VANETs. Critical reviews were received from S. Olariu,
G. Yan, S. El-Tawab (Old Dominion University, USA), C. M. Pinotti (University of Perugia, Italy), S. Salleh (University of Technology, Malaysia), M. E. Rizvi and S. Zehra (Norfolk State University, USA), Z. Zaidi (NICTA, Australia), and X. Chen (University of Oklahoma, USA).

In "Spectrum sensing for cognitive vehicular networks over composite fading," the authors consider shared utilization of the radio spectrum via cognitive radio systems to increase spectrum efficiency and quality of vehicular services. A cognitive radio system is a mechanism which allows unlicensed cognitive users (CUs) to utilize idle unused bands. Spectrum sensing is the first step that should be carried out before permitting cognitive clients to approach an authorized channel. A viable choice for spectrum sensing due to its simplicity, low computational cost, and ability to be applied on any kind of deterministic signal is energy detection (ED). However, hidden terminal and low SNR problems due to shadowfading put fundamental limits to the sensing performance and practical entailments in designing of cognitive vehicular networks. Extensive modeling efforts are then being carried out to cope with varying channel characteristics, particularly multipath fading and shadowing. In this paper, the authors examine the performance of spectrum sensing using ED over Gamma-shadowed Nakagamim composite fading channel to cater both small and large-scale fading. The results highlight the notable impact of shadowing spread and fading severity on detection performance.

In "Disseminating large amount of data to vehicular network in an urban area," the problem of distributing large amount of data from multiple sources in an urban area is 
investigated. The authors have shed light on an opportunistic approach for information collection in which a vehicle obtains information about resources from encountered vehicles. Due to the highly dynamic nature of the underlying vehicular network topology, reliable dissemination is introduced from multiple sources when each node in the network shares a limited amount of its resources for cooperating with others. By using rateless coding at the road side unit (RSU) and using vehicles as data carriers, an efficient way to achieve reliable dissemination to all nodes is described.

Recently, the IntelliDriveSM initiative has been proposed by the US Department of Transportation (USDOT) to enhance on-road safety and efficiency. In "The effect of intellidrive on the efficiency of highway transportation systems," a mathematical framework which predicts the effect of IntelliDrive on the efficiency of multilane highway systems prior to their real-life deployment is covered. The authors have shown that intervehicular communications improves the flow of vehicles by reducing the perception-reaction (P$\mathrm{R})$ times of drivers and, in some cases, allowing for more efficient lane-changing operations.

The paper "Heterogeneous wireless sensor network for transportation system applications," outlines the author's experience in the Embedded Middleware in Mobility Applications (EMMA) project and provides an illustration of the important role that wireless sensor technology can play in future transport system. The paper presents the employment of heterogeneous sensors to develop transportation system applications and focuses on how cooperation between vehicle and infrastructure can be addressed. It also presents encouraging results obtained from the experiments in investigating the feasibility of utilizing wireless sensor in vehicle and vehicle-to-infrastructure communication in real transportation applications.

In "Evaluation of selective broadcast algorithms for safety applications in vehicular ad hoc networks," evaluation focusing on the performance with respect to safety, rather than to classical network aspects like throughput, loss, and delay is presented. In this research, four new performance criteria are defined to address the effectiveness, efficiency, timeliness, and overhead of the candidate broadcast algorithms in terms of safety warning delivery. Four different selective broadcast algorithms used for information dissemination in VANETs are simulated. The results obtained help the readers to understand better the design requirements of a highperformance selective broadcast algorithm.

In "Time in privacy preserving LBSs: an overlooked dimension," privacy issues in location-based services (LBSs), that is, traffic monitoring, congestion-based, and "pay-asyou-go" road pricing, are considered. The main privacy threat in such services is the possibility to identify the user that requests a given service and his/her location at the time of the request. To specify a level of anonymity, a feelingbased model has been recently introduced which allows a user to define his/her desired level of anonymity simply by specifying a given area (e.g., a shopping mall). The entropy of the selected area is used to describe the area's popularity. In turn, the popularity is expressed in terms of footprints of the visitors-these being a representation of the amount of time a user spends in a given area-in the selected area. While this privacy model is claimed to be independent from the specific knowledge of the adversary about users' footprints, in this paper, the authors argue that an adversary that has a more structured knowledge over time can pose a threat to the privacy guarantees of the model. The claim is supported with both analysis and a concrete example on the mobility traces of cabs of San Francisco.

\section{Acknowledgment}

We are very pleased and thankful to receive the brilliant contributions and supports from many distinguished authors and organizations all over the world. I give my sincerest appreciation to Ghada Ali, Hindawi Publishing Corporation, for her timely support, excellent advice, and kind words of encouragement. Without her support, this special issue certainly would not have been possible. I thank the authors, all members of the Editorial Board, and members of publishing team in Hindawi Publishing Corporation for their efforts in providing significant supports and suggestions and organizing this special issue from ground zero.

Syed R. Rizvi Stephan Olariu Cristina M. Pinotti Shaharuddin Salleh Mona E. Rizvi Zainab Zaidi 

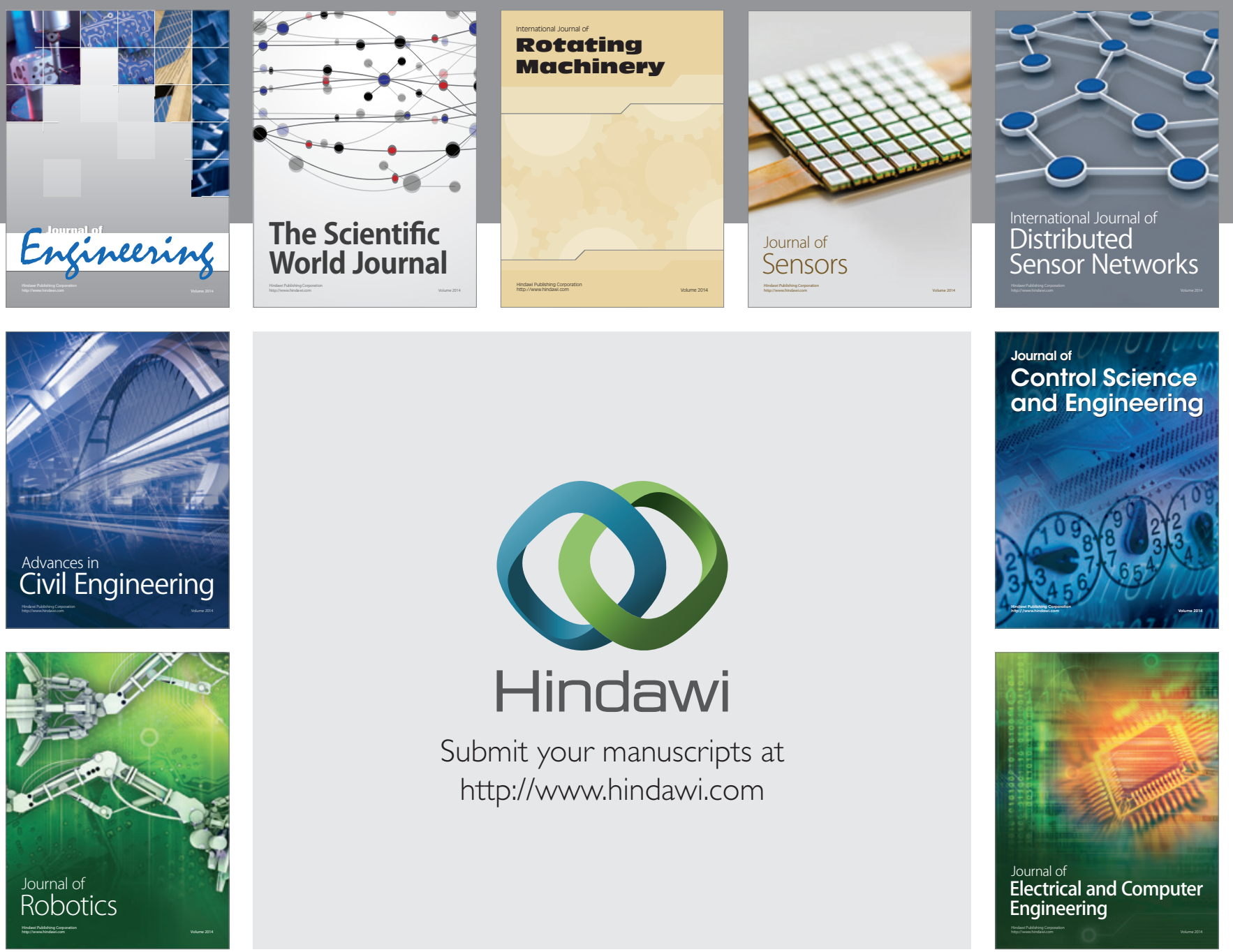

Submit your manuscripts at

http://www.hindawi.com
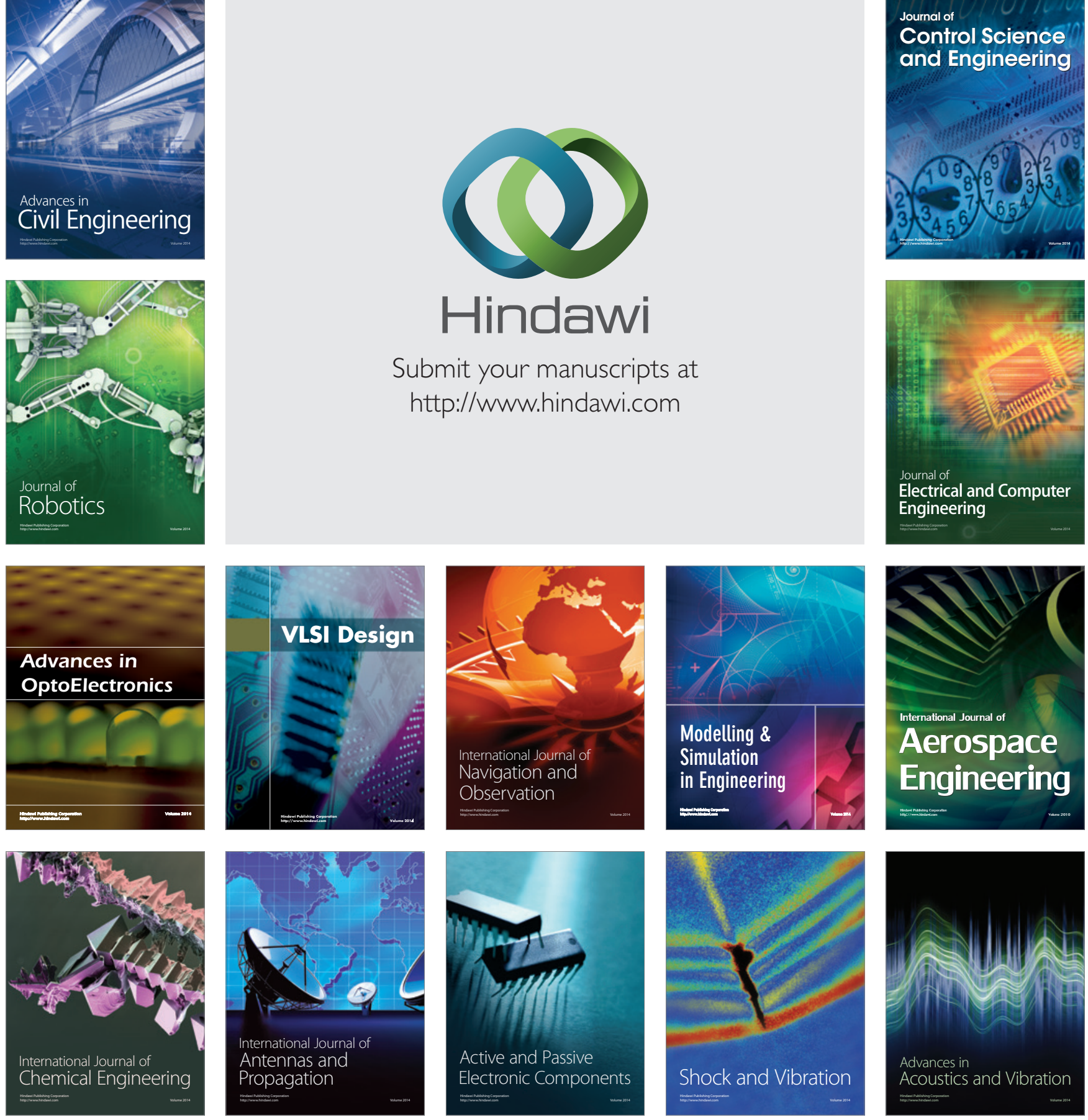